\title{
Effect of He-Ne laser radiation on healing of osteochondral defect in rabbit: A histological study
}

\author{
Mohammad Bayat, PhD; ${ }^{\text {* }}$ Farshad Javadieh; ${ }^{2}$ Masoomeh Dadpay, MD $^{3}$ \\ ${ }^{1}$ Cellular and Molecular Biology Research Center, Medical Faculty, Shahid Beheshti University, MC, Tehran, Iran; \\ ${ }^{2}$ Medical Faculty, Shahid Beheshti University, MC, Tehran, Iran; ${ }^{3}$ Pathology Department, 501 Army Hospital, Tehran, Iran
}

\begin{abstract}
This study examined the influence of low-level laser therapy (LLLT) on the healing of a large osteochondral defect in rabbits. An osteochondral defect with $5 \mathrm{~mm}$ diameter was surgically induced in the right femoral patellar groove of 48 adult male rabbits. They were divided into a control and an experimental group. The rabbits were treated at 2, 4, 8, and 16 weeks after surgery, with six rabbits in each study period being tested at each biweekly period. The experimental group received LLLT with a helium-neon (He-Ne) laser $(632.8 \mathrm{~nm}$, $10 \mathrm{~mW}$ ) of $148.4 \mathrm{~J} / \mathrm{cm}^{2}$ three times a week, and the control group received placebo LLLT with equipment switched off. The defects were examined macroscopically and microscopically. The results of the histological examination 2 weeks after surgery showed that the osteochondral healing of the control group was significantly accelerated compared with that of the experimental group. However, the osteochondral healing of the experimental group 4 weeks and 16 weeks after surgery showed that healing accelerated significantly compared with that of the control group. The conclusion was that LLLT with an He-Ne laser could not significantly accelerate healing of a large osteochondral defect in rabbits of the experimental group compared with that of the control group throughout the duration of the present study.
\end{abstract}

Key words: articular cartilage, He-Ne laser, histology, histomorphometry, knee, laser radiation, low-level laser therapy, osteochondral defect, rabbit, wound healing.

\section{INTRODUCTION}

Articular cartilage has a simple architecture composed of a unique type of cell, i.e., the chondrocyte, embedded in a dense extracellular matrix (ECM). Both the chondrocyte and ECM distribute within successive cartilage layers identified as superficial, transitional, radial, and calcified zones. The subchondral bone is located deep in the calcified zone. Articular chondrocytes in adults do not divide but help maintain the integrity of the cartilage through balanced synthetic and catabolic activities [1]. Cartilage lesions are classified as either full or partial thickness, depending on whether or not they extend to the subchondral bone [2]. Historically, articular cartilage has been considered to have only a weak capacity for repair, as reported by Hunter 250 years ago [3]. Researchers have generally accepted that those defects that penetrate the subchondral bone (full-thickness defects) are repaired with various tissues, from a fibrous tissue to fibrocartilage. However, the reparative tissue, even histologically hyaline-like cartilage, lacks the biomechanical capabilities to express some cartilage-specific molecules, and its

\footnotetext{
Abbreviations: $\mathrm{AED}=$ architecture within entire defect, $\mathrm{ECM}=$ extracellular matrix, FD = filling of the defect, IRT = integration of repaired tissue, He-Ne = helium-neon, LLLT = low-level laser therapy, MS = matrix staining, TEM = transmission electron microscopic, THP = total histological parameter.

*Address all correspondence to Dr. Mohammad Bayat, Cellular and Molecular Biology Research Center, Medical Faculty, Shahid Beheshti University, MC, PO Box 19395/ 4719, Tehran, 1985717443, Iran; +98-21-2387-2502; fax: +9821-2243-9949. Email: bayat m@yahoo.com

DOI:10.1682/JRRD.2009.03.0035
} 
biomechanical durability is inferior to that of agematched normal articular cartilage [4]. A number of attempts have been made to develop clinically useful procedures for repairing damaged articular cartilage. The term low-level laser therapy (LLLT) is broadly applied to the therapeutic effects of laser. The clinical application of LLLT is growing rapidly, and several review articles on clinical applications of LLLT have been published [5]. LLLT with a helium-neon (He-Ne) laser has been used in treating musculoskeletal complications [6-11]. In this regard, research has reported that LLLT with an He-Ne laser can improve immobilized articular cartilage and damaged articular cartilage in vivo [6-9] and that LLLT can increase the number of chondrocytes in vitro [10]. Very recently, Peccin et al. demonstrated that an He-Ne laser induces cartilage neoformation after 40 days following therapy [11]. Since no reports have been published on the effect of a low-level He-Ne laser with a wavelength of $632.8 \mathrm{~nm}$ on healing a large osteochondral defect in rabbits, this study examines the potential therapeutic effect of LLLT with an He-Ne laser on the histological parameters of healing a large osteochondral defect in rabbits.

\section{METHODS}

\section{Animal Procedures}

We used 48 adult white New Zealand rabbits aged 7 months and weighing 2,000 $\pm 300 \mathrm{~g}$ (mean \pm standard deviation) at the beginning of the experiment. (Note that subsequent values are expressed as mean \pm standard deviation unless specified otherwise.) All animals were fed a standard diet with tap water ad libitum. Rabbits were randomly divided into a control group $(n=24)$ and an experimental group $(n=24)$, with four subgroups of six rabbits in each group. The rabbits were treated at 2, 4, 8 , and 16 weeks in the experimental and control groups. All animal care, as well as experimental procedures, was conducted in accordance with institutional guidelines. Surgery was performed on all rabbits under sterile conditions. Anesthesia was achieved by intramuscular injection of $50 \mathrm{mg} / \mathrm{kg}$ Ketamine and $5 \mathrm{mg} / \mathrm{kg}$ diazepam. A medial parapatellar incision was made in the right knee joint of each rabbit. We dislocated the patella laterally to expose the articular surface of the patellar groove of femur bone. One circular defect, $5 \times 10 \mathrm{~mm}$, was drilled through the articular cartilage into the bleeding subchondral bone in the distal part of the patellar groove with the use of a lowspeed dental drill (Dental Fabriktreffurt, Delab; Treffurt, Germany). The joint capsule and skin were closed by 03 catgut and 04 nylon sutures, respectively. Antibiotic therapy with ceftrax (Jaber ben Hayyan; Tehran, Iran) at a dose of $50 \mathrm{mg} / \mathrm{kg}$ was administered immediately before operation and at 24 and 48 hours after surgery. All animals were allowed to walk freely without any splint.

\section{Low-Level Laser Therapy}

One point of each right and left femoral condyle of the right knee of the rabbits in the experimental group was exposed by a laser immediately after surgery. The specifications of the laser used (Iranian Atomic Energy Organization; Bonab, Iran) were shown as-

- Laser source: $10 \mathrm{~mW}$ He-Ne laser.

- Wavelength: $632.8 \mathrm{~nm}$.

- Frequency: Continuous.

- Surface area of laser beam: $0.0314 \mathrm{~cm}^{2}$.

- Timing: $466 \mathrm{~s}$.

- Energy density: $148.4 \mathrm{~J} / \mathrm{cm}^{2}$.

The rabbits of the experimental group were relaxed in a restrainer by injection of one-half dose of anesthetized drugs during laser irradiation. Based on the side effects of daily general anesthesia on rabbits, such as general body weakness and poor healing of wounds, that we observed in our pilot study, we performed LLLT three times a week in the current study. In our previous studies, the He-Ne laser showed positive effects on immobilized articular cartilage. So in the present study, we used the laser parameters of the studies just mentioned $[6,9]$.

We found no methodological differences between the control and experimental groups except the use of LLLT. The rabbits in the control group were held in a restrainer, received one-half dose of anesthetizing drugs, and received placebo LLLT with switched-off laser equipment, so no differences existed between control and experimental groups except LLLT.

\section{Histological Examination}

The rabbits were killed with inhalation of chloroform in a closed space at 2 weeks, 4 weeks, 8 weeks, and 16 weeks after surgery. The number of rabbits in each experimental study period for both study groups was six. We photographed defects using a Canon PowerShot G6 (Tokyo, Japan) and grossly examined and macroscopically evaluated them using a macroscopic grading system (Table 1). 
The distal part of femur was cut from the bone, and the defects were fixed in 10 percent neutral buffered formalin, decalcified in ethylenediaminetetra-acetic acid solution, and embedded in paraffin for examining histologically. We cut sections sagittally from the proximal to distal aspect of the patellar groove, stained them with hematoxylin and eosin and 0.2 percent alcoholic solution of toluidine blue, and evaluated them by using a histological grading scale (Table 2) [12-13]. The histological analysis was performed under light microscopy. Two experts blinded to the treatment of each group independently examined each specimen. In case of disagreement, examiners reevaluated the specimen and reached a consensus. The slides were evaluated before each group was identified.

\section{Statistical Analysis}

We performed a student $t$-test to determine the differences between the control and experimental groups. The statistically significant difference was $p<0.05$.

\section{RESULTS}

\section{Macroscopic Appearance of Osteochondral Defects}

The mean of the surface appearance and the filling of the defect (FD) of the experimental group was lower (better) than the control group at sequential intervals (2, 4, 8, and 16 wk periods) in most cases (5/8) (Figure 1). However, student $t$-test showed that the FD was significantly lower in the control group $(1 \pm 0)$ than in the experimental group $(2.16 \pm 0.75) 2$ weeks after surgery $(p=0.013)$.

Table 1.

Scoring system for macroscopic appearance of osteochondral defect in articular cartilage of rabbits $(N=48)$.

\begin{tabular}{lc}
\hline \multicolumn{1}{c}{ Category } & $\begin{array}{c}\text { Score } \\
\text { (Points) }\end{array}$ \\
\hline Surface Appearance Normal, Smooth & 0 \\
Hyaline (\%) & 1 \\
$>75$ & 2 \\
From 50-75 & 3 \\
$<50$ & \\
Filling of Defect (\%) & 0 \\
100 & 1 \\
$>75$ & 2 \\
From 50-75 & 3 \\
$<50$ & 3 \\
\hline \hline
\end{tabular}

Table 2.

Modified histological grading scale for osteochondral defects of articular cartage of rabbits $(N=48)$.

\begin{tabular}{lc}
\hline \multicolumn{1}{c}{ Category } & $\begin{array}{c}\text { Score } \\
\text { (Points) }\end{array}$ \\
\hline $\begin{array}{l}\text { Filling of Defect Relative to Surface of Normal } \\
\text { Adjacent Cartilage (\%) }\end{array}$ \\
111-123 & \\
$91-110$ & 1 \\
$76-90$ & 0 \\
$51-75$ & 1 \\
$26-50$ & 2 \\
$<25$ & 3 \\
\end{tabular}

Integration of Repaired Tissue with Surrounding Articular Cartilage

Normal continuity and integration $\quad 0$

Decreased cells 1

Gap or lack of continuity on one side 2

Gap or lack of continuity on both sides 3

Matrix Staining with Toluidine Blue

Normal 0

Slightly reduced $\quad 1$

Moderately reduced 2

Substantially reduced 3

None 4

Cellular Morphology*

Normal 0

Mostly round cells with morphology of chondrocytes

$>75 \%$ of tissue with columns in 1 radial zone

$25 \%-75 \%$ of tissue with columns in $\quad 1$ radial zone

$<25 \%$ of tissue with columns in radial zone (disorganized)

$50 \%$ round cells with morphology of chondrocytes

$>75 \%$ of tissue with columns in radial zone

$25 \%-75 \%$ of tissue with columns in 3 radial zone

$<25 \%$ of tissue with columns in 4 radial zone (disorganized)

Mostly spindle-shaped (fibroblast-like) 5 cells

Architecture Within Entire Defect

(Not Including Margins)

Normal

0

1-3 small voids 
Table 2. (Continued)

Modified histological grading scale for osteochondral defects of articular cartage of rabbits $(N=48)$.

\begin{tabular}{|c|c|}
\hline Category & $\begin{array}{c}\text { Score } \\
\text { (Points) }\end{array}$ \\
\hline 1-3 large voids & 2 \\
\hline >3 large voids & 3 \\
\hline Clefts for fibrillations & 4 \\
\hline \multicolumn{2}{|l|}{ Architecture of Surface } \\
\hline Normal & 0 \\
\hline Slight fibrillation or irregularity & 1 \\
\hline Moderate fibrillation or irregularity & 2 \\
\hline Severe fibrillation or disruption & 3 \\
\hline \multicolumn{2}{|l|}{ New Subchondral Bone (\%) } \\
\hline \multicolumn{2}{|l|}{ Below original $\mathrm{TM}^{\dagger}$} \\
\hline $90-100$ & 0 \\
\hline $75-89$ & 1 \\
\hline $50-74$ & 2 \\
\hline $25-49$ & 3 \\
\hline$<25$ & 4 \\
\hline \multicolumn{2}{|l|}{ Above original $\mathrm{TM}^{\ddagger}(\%)$} \\
\hline $90-100$ & 0 \\
\hline $75-89$ & 1 \\
\hline $50-74$ & 2 \\
\hline $25-49$ & 3 \\
\hline$<25$ & 4 \\
\hline \multicolumn{2}{|l|}{ Formation of TM (\%) } \\
\hline Complete & 0 \\
\hline 75-99 & 1 \\
\hline $50-74$ & 2 \\
\hline $25-49$ & 3 \\
\hline$<25$ & 4 \\
\hline
\end{tabular}

${ }^{*}$ Determined in cartilage above original tidemark (TM).

${ }^{\dagger} 100 \%$ indicates that new bone is at same level as original TM.

${ }_{100} \%$ indicates that thickness of repair cartilage is same as that of normal adjacent cartilage.

\section{Histological Grading Scale of Osteochondral Defect}

Histological micrographs of repairing tissue in study groups at sequential intervals (2, 4, 8, and 16 wk periods) are shown in Figure 2. The mean of the histological grading scale of the experimental group was lower (better) in most cases (19/23).

\section{2-Week Study Period}

No significant differences were found among most of the histological parameters between the control and experimental groups. However, the total histological grading scale $(3.83 \pm 0.40)$ of the experimental group was sig-

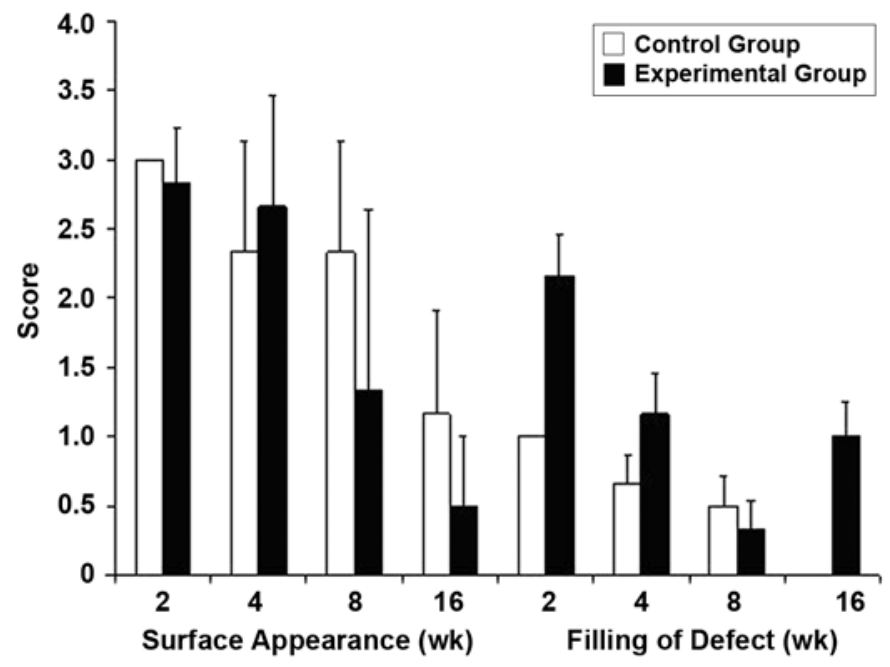

Figure 1.

Mean \pm standard deviation of scoring system for macroscopic appearance of osteochondral defect of articular cartilage of study groups at sequential intervals. Student $t$-test revealed that filling of defect was significantly lower between control and experimental groups $(p=0.013)$.

nificantly higher than that of the control group (3.16 \pm $0.40)(p=0.018)$.

\section{4-Week Study Period}

The mean of each integration of repaired tissue (IRT) (2.00 \pm 1.54$)$, matrix staining (MS) $(3.66 \pm 0.81)$, and architecture within entire defect (AED) $(4.0 \pm 0.0)$ of the experimental group was significantly lower than the mean of each IRT $(1.33 \pm 1.50)$, MS $(1.33 \pm 1.75)$, and AED $(1.33 \pm 2.00)$ of the control group; $p$-values = $0.005,0.021$, and 0.025 , respectively).

\section{8-Week Study Period}

No significant differences were found among all the histological parameters and the total histological parameters (THPs) between the control and experimental groups.

\section{6-Week Study Period}

Most of the histological parameters and THPs of the experimental group increased significantly compared with those of the control group. The mean values of FD, IRT, MS, cellular morphology, AED, architecture of surface, and THPs of the experimental group were significantly lower than the mean values of those of the control group (Table 3). However, the tidemark $(3.83 \pm 0.40)$ of the experimental group was significantly higher than that of the control group $(1.66 \pm 1.21)(p=0.006)$. 


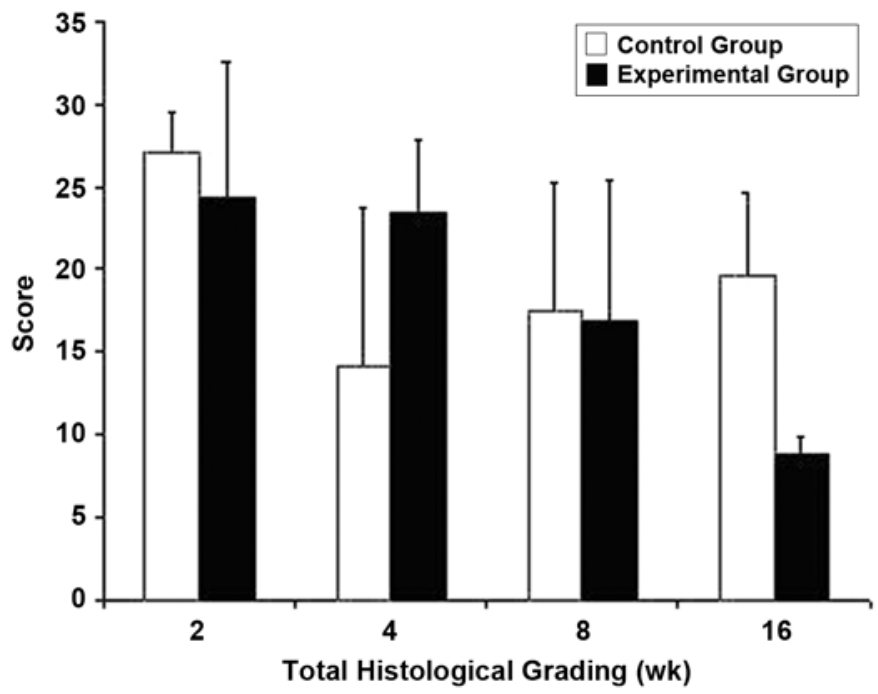

Figure 2.

Mean \pm standard deviation of scoring system for total histological scores of osteochondral defect of articular cartilage of study groups at sequential intervals. Student $t$-test showed (1) total histological parameter (THP) $(3.83 \pm 0.40)$ of experimental group was significantly higher than that of control group $(3.16 \pm 0.40) 2$ wk after surgery ( $p=0.018)$; (2) mean of integration of repaired tissue (IRT) $(2.00 \pm$ 1.54), matrix staining (MS) (3.66 \pm 0.81$)$, and architecture within entire defect (AED) $(4.0 \pm 0)$ of experimental group was significantly lower than mean of control group (IRT, $1.33 \pm 1.50$; MS, $1.33 \pm 1.75$; and AED, $1.33 \pm 2.00$, respectively) 4 wk after surgery ( $p=0.005, p=$ 0.021 , and $p=0.025$, respectively); (3) most histological grading scales and THPs of experimental group were significantly higher than those of control group 16 wk after surgery, and mean of THP (8.83 \pm 0.98) of experimental group was significantly lower than that of control group (19.66 $\pm 4.00, p=0.003)$. However, at same time, tidemark $(3.83 \pm 0.40)$ of experimental group was significantly higher than that of control group $(1.66 \pm 1.21)(p=0.006)$.

\section{DISCUSSION}

The results of the current study could not show a positive effect of LLLT with the He-Ne laser on healing of one large osteochondral defect in rabbits throughout the study duration.

In two recent studies from the current authors' laboratory, the capability of He-Ne laser biostimulation to neutralize adverse effects of knee joint immobilization in rabbits was investigated [6,9]. In these studies, the researchers divided the rabbits into three groups:

- Experimental group, which received LLLT with $13 \mathrm{~J} /$ $\mathrm{cm}^{2}$ (without considering the beam area of the laser used for calculating energy density) three times a week after immobilization of right knee.
Table 3.

Mean \pm standard deviation of scoring scale for total histological scores of osteochondral defect of articular cartilage of study groups at 16-week study period.

\begin{tabular}{lcrc}
\hline Variable & Experimental & Control & $\boldsymbol{p}$-Value \\
\hline FD & $0.0 \pm 0.0$ & $1.33 \pm 1.03$ & 0.025 \\
IRT & $0.0 \pm 0.0$ & $2.33 \pm 0.51$ & $<0.001$ \\
MS & $0.16 \pm 0.40$ & $2.66 \pm 1.36$ & 0.025 \\
CM & $0.0 \pm 0.0$ & $2.66 \pm 1.50$ & 0.001 \\
AED & $0.0 \pm 0.0$ & $3.67 \pm 0.82$ & $<0.001$ \\
AS & $0.0 \pm 0.0$ & $2.80 \pm 0.98$ & 0.001 \\
THP & $8.83 \pm 0.98$ & $19.66 \pm 4.00$ & 0.003 \\
TM & $3.83 \pm 0.40$ & $1.66 \pm 1.21$ & 0.006 \\
\hline
\end{tabular}

$\overline{\mathrm{AED}}=$ architecture within entire defect, $\mathrm{AS}=$ architecture of surface, $\mathrm{CM}=$ cellular morphology, $\mathrm{FD}=$ filling of defect, IRT = integration of repaired tissue, MS = matrix staining, THP = total histological parameter, TM = tidemark.

- Control group, which did not receive laser therapy after immobilization.

- Normal group, which received neither immobilization nor LLLT.

Light microscopic and transmission electron microscopic (TEM) examinations were performed after 4- and 7-week immobilization periods [6] and a 13-week immobilization period [9]. In the first study, results showed that the depths of the chondrocytes filopodia and the articular cartilage in the laser-treated group were significantly higher than those of the control group. In the second study, TEM examination revealed marked filopodia and euchromatin nucleus in the experimental group [9]. The number of chondrocytes and depth of articular cartilage of the experimental group also increased significantly compared with that of the control group.

Very recently, Peccin et al. demonstrated that the HeNe laser is capable of inducing cartilage neoformation [11]. They induced the bilateral knee cartilage defects in rabbits using a spherical bur. Those with the right knee defect served as the control group. Peccin et al. reported that after 40 days, a well-organized fibrous tissue fully filled the lesions of the control group, whereas the He-Ne laser-treated damages showed that a hyaline cartilage filled the lesion completely. Apparently, in the abovementioned studies, laser penetration was so deep that it could biostimulate chondrocytes of articular cartilage [6,9] and damage cartilage neoformation [11].

The current study results contrast with the Peccin et al. study results [11]. Peccin et al. used internal controls. However, systemic effect of LLLT has been reported in previous studies [14-15]. In this regard, Khadra et al. have 
reported that LLLT can stimulate the release of substances such as growth factors and cytokines into circulatory systems, so the laser could affect the untreated side of experimental animals or subjects [16]. On the other hand, Peccin et al. damaged the articular cartilage, whereas in the current study, the subchondral bone was deeply damaged. However, the present study results showed that LLLT did not accelerate osteochondral repair compared with that of the control group. Apparently, the results of our previous study [17], which reported a significant increase in repairing osteochondral defect using an infrared laser, were superior to the results of present study. Comparison of the present study results with our previous study [17] results suggests that the infrared laser with longer wavelength penetrates more deeply to repair tissue than the He-Ne laser with shorter wavelength [18]. We speculate that the He-Ne laser did not penetrate the articular cartilage and subchondral bone deep enough to biostimulate acceleration of osteochondral healing as the infrared laser did. Results of the current study comply with the results of Calatrava et al. [19]. Calatrava et al. induced a lesion on the hind limb of normal rabbits. These animals were divided into two groups. One group was treated with an He-Ne laser, and the other was treated with an infrared laser. At the end of treatment, a histological examination showed hyaline cartilage in the infrared laser group, fibrocartilage in the He-Ne laser group, and granulation tissue in limbs of the control group. Calatrava et al. concluded that the infrared laser significantly accelerated the articular cartilage healing process in the defect compared with that of the HeNe laser [19].

\section{CONCLUSION}

We conclude that LLLT with an He-Ne laser of one large osteochondral defect in rabbits of the experimental group could not significantly accelerate healing of a large osteochondral defect compared with that of the control group throughout the duration of the present study.

\section{ACKNOWLEDGMENTS}

\author{
Author Contributions: \\ Study concept and design: M. Bayat. \\ Acquisition of data: M. Bayat, F. Javadieh. \\ Analysis and interpretation of data: M. Bayat, F. Javadieh, \\ M. Dadpay. \\ Drafting of manuscript: M. Bayat.
}

Critical revision of manuscript for important intellectual content:

M. Bayat.

Obtained funding: M. Bayat.

Administrative, technical, or material support: M. Bayat.

Study supervision: M. Bayat.

Financial Disclosures: The authors have declared that no competing interests exist.

Funding/Support: This material was based on work supported by the Vice-Chancellor of Research of Shahid Beheshti University, MC, grant 3/5740.

Additional Contributions: We wish to thank the late Mrs. Jamileh Rezaei. We also extend our thanks to the Vice-Chancellor of Research in the Shahid Beheshti University, MC, for financial support (grant 3/ 5740) and Miss Nasrin Khaterie for drawing the figures.

\section{REFERENCES}

1. Cancedda R, Dozin B, Giannoni P, Quarto R. Tissue engineering and cell therapy on cartilage and bone. Matrix Biol. 2003;22(1):81-89. [PMID: 12714045]

DOI:10.1016/S0945-053X(03)00012-X

2. Beris AE, Lykissas MG, Papageorgiou CD, Georgoulis AD. Advances in articular cartilage repair. Injury. 2005; 36(Suppl 4):S14-23. [PMID: 16291320] DOI:10.1016/j.injury.2005.10.007

3. Hunter W. On the structure and disease of articulating cartilages. 1743. Clin Orthop Relat Res. 1995;(317):3-6. [PMID: 7671493]

4. Hunziker EB. Articular cartilage repair: Basic science and clinical progress. A review of the current status and prospects. Osteoarthritis Cartilage. 2002;10(6):432-63.

[PMID: 12056848] DOI:10.1053/joca.2002.0801

5. Reddy GK. Photobiological basis and clinical role of lowintensity lasers in biology and medicine. J Clin Laser Med Surg. 2004;22(2):141-50. [PMID: 15165389] DOI:10.1089/104454704774076208

6. Bayat M, Ansari A, Hekmat H. Effect of low-power helium-neon laser irradiation on 13-week immobilized articular cartilage of rabbits. Indian J Exp Biol. 2004;42(9): 866-70. [PMID: 15462178]

7. Lin YS, Huang MH, Chai CY, Yang RC. Effects of heliumneon laser on levels of stress protein and arthritic histopathology in experimental osteoarthritis. Am J Phys Med Rehabil. 2004;83(10):758-65. [PMID: 15385784] DOI:10.1097/01.PHM.0000137310.15943.19

8. Lin YS, Huang MH, Chai CY. Effects of helium-neon laser on the mucopolysaccharide induction in experimental osteoarthritic cartilage. Osteoarthritis Cartilage. 2006;14(4):377-83. [PMID: 16359876] DOI:10.1016/j.joca.2005.10.010

9. Bayat M, Ansari E, Gholami N, Bayat A. Effect of low-level helium-neon laser therapy on histological and ultrastructural 
features of immobilized rabbit articular cartilage. J Photochem Photobiol B. 2007;87(2):81-87.

[PMID: 17407820]

DOI:10.1016/j.jphotobiol.2007.02.002

10. Jia YL, Guo ZY. Effect of low-power He-Ne laser irradiation on rabbit articular chondrocytes in vitro. Lasers Surg Med. 2004;34(4):323-28. [PMID: 15083493]

DOI:10.1002/lsm.20017

11. Peccin MS, da Silva PRG, Renno AC, Ribeiro DA. Lowenergy helium-neon laser ensures knee cartilage repair: An experimental short-term assay in rabbits. J Laser Appl. 2008; 20(3):165-68.

12. Sellers RS, Peluso D, Morris EA. The effect of recombinant human bone morphogenetic protein-2 (rhBMP-2) on the healing of full-thickness defects of articular cartilage. J Bone Joint Surg Am. 1997;79(10):1452-63. [PMID: 9378731]

13. Pineda S, Pollack A, Stevenson S, Goldberg V, Caplan A. A semiquantitative scale for histologic grading of articular cartilage repair. Acta Anat (Basel). 1992;143(4):335-40. [PMID: 1502876] DOI:10.1159/000147272

14. Rochkind S, Rousso M, Nissan M, Villarreal M, Barr-Nea L, Rees DG. Systemic effects of low-power laser irradiation on the peripheral and central nervous, cutaneous wound, and burns. Lasers Surg Med. 1989;9(2):174-82.

[PMID: 2716462]

DOI:10.1002/lsm.1900090214
15. Schindl A, Heinze G, Schindl M, Pernerstorfer-Schön H, Schindl L. Systemic effects of low-intensity laser irradiation on skin microcirculation in patients with diabetic microangiopathy. Microvasc Res. 2002;64(2):240-46.

[PMID: 12204648]

DOI:10.1006/mvre.2002.2429

16. Khadra M, Kasem N, Haanaes HR, Ellingsen JE, Lyngstadaas SP. Enhancement of bone formation in rat calvarial bone defects using low-level laser therapy. Oral Surg Oral Med Oral Pathol Oral Radiol Endod. 2004;97(6):693-700.

[PMID: 15184850]

DOI:10.1016/j.tripleo.2003.11.008

17. Kamali F, Bayat M, Torkaman G, Ebrahimi E, Salavati M. The therapeutic effect of low-level laser on repair of osteochondral defects in rabbit knee. J Photochem Photobiol B. 2007;88(1):11-15. [PMID: 17555980]

DOI:10.1016/j.jphotobiol.2007.04.010

18. Cameron MH, Perez D, Otaho-Lata S. Electromagnetic fields. In: Cameron MH, editor. Physical agents in rehabilitation: From research to practice. St. Louis (MO): W. B. Saunders; 2003. p. 303-44.

19. Calatrava IR, Valenzuela JM, Gómez-Villamandos RJ, Redondo JI, Gómez-Villamandos JC, Jurado IA. Histological and clinical response of articular cartilage to low-level laser therapy: Experimental study. Lasers Med Sci. 1997; 12:117-21.

Submitted for publication March 28, 2009. Accepted in revised form September 1, 2009. 
